\title{
Probabilidade de Erro Bit em Receptor MRC com Modulação QAM sob Desvanecimento Generalizado: Parte I
}

\author{
Wamberto J. L. Queiroz, Marcelo S. Alencar e Francisco Madeiro
}

\begin{abstract}
Resumo-Este artigo apresenta um tratamento matemático unificado para a avaliação da probabilidade de erro de bit dos esquemas de modulação M-PAM, M-QAM e R-QAM sob desvanecimento generalizado. As expressões obtidas para a BEP são escritas em termos da função geratriz de momentos da SNR por bit para cada ramo do receptor utilizado. Essa função geratriz de momentos varia de acordo com o tipo de desvanecimento considerado. São assumidos um receptor com razão máxima de combinação e codificação Gray para os bits modulados.
\end{abstract}

Palavras-Chave-Receptor MRC, desvanecimento generalizado, M-PAM, M-QAM, R-QAM.

Abstract-This article presents a unified mathematical treatment to the evaluation of the bit error probability (BEP) of the M-PAM, M-QAM and R-QAM modulation schemes, subject to generalized fading. The expressions obtained for the BEP are written in terms of the moment generating function of the signalto-noise ratio per bit for each branch of the used receiver. That moment generating function varies in accordance with the type of fading considered. A maximum combining ratio receiver and Gray encoding for the modulated bits are assumed.

Keywords-MRC receiver, generalized fading, M-PAM, MQAM, R-QAM.

\section{INTRODUÇÃO}

A maioria dos sistemas de comunicações sem fio modernos tem seus sinais de informação constantemente submetidos a diferentes formas de desvanecimento, capazes de degradar seriamente a qualidade dos enlaces, caso soluções apropriadas não sejam adotadas. Uma das técnicas propostas para diminuir os efeitos do desvanecimento é a recepção com diversidade espacial, obtida pelo uso de arranjos de elementos de antenas igualmente espaçados, associados a um receptor que combina diversas réplicas recebidas de um mesmo sinal e maximiza sua relação sinal-ruído (SNR - Signal to Noise Ratio) de saída.

Essa estrutura de recepção, conhecida como receptor com razão máxima de combinação (MRC - Maximal Ratio Combiner), tem sido proposta por vários autores, associada a esquemas de modulação coerente em ambientes com desvanecimento Rayleigh e Nakagami [1]-[3]. Uma análise unificada da probabilidade de erro de símbolo (SEP - Symbol Error Probability) de esquemas de modulação coerente, sob diferentes formas de desvanecimento, foi apresentada em [4],

Wamberto José Lira Queiroz, Universidade Federal do Ceará, Sobral, Brasil, wamberto@ufc.br. Marcelo Sampaio de Alencar, Universidade Federal de Campina Grande, Campina Grande, Brasil, malencar@dee.ufcg.edu.br. Francisco Madeiro, Universidade de Pernambuco, Recife, Brasil, franciscomadeiro@yahoo.com.br. Os autores também estão com o Instituto de Estudos Avançados em Comunicações (Iecom), Campina Grande, Brasil. contendo inclusive expressões para o esquema M-QAM ( $M$ ary Quadrature Amplitude Modulation).

Antes de 2002, qualquer avaliação do efeito do desvanecimento nos esquemas M-QAM, M-PAM (M-ary Pulse Amplitude Modulation) e R-QAM (Rectangular Quadrature Amplitude Modulation) só podia ser feita por meio das aproximações obtidas, até então, para a probabilidade de erro de bit desses esquemas sob o ruído aditivo gaussiano branco (AWGN Additive White Gaussian Noise). Em 2002 uma importante contribuição obtida por K. Cho e D. Yoon [5] forneceu uma expressão generalizada para o cálculo da probabilidade de erro de bit (BEP - Bit Error Probability) para os equemas M-QAM (quadrado) e R-QAM (retangular) sob ação do ruído AWGN e mapeamento por código Gray para as seqüências de bits. A partir desse resultado de Cho e Yoon, pode-se obter expressões mais gerais para a BEP dos esquemas de modulação citados, sob diferentes formas de desvanecimento, que englobem as contribuições fornecidas por meio de aproximações para a BEP dos esquemas M-QAM sob ruído AWGN.

Algumas dimensões de constelação M-QAM e R-QAM têm sido bastante utilizadas em implantações práticas de sistemas de comunicações por permitirem considerável aumento da taxa de transmissão sem aumento da largura de faixa ocupada. Devido a essa característica, as pesquisas que visam avaliar o desempenho de esquemas QAM em modelos de transmissão sem fio continuam despertando interesse. Nas referências [6] e [7], por exemplo, são apresentadas expressões para a probabilidade de erro de símbolo (SEP - Symbol Error Probability) do sistema QAM retangular em desvanecimento Nakagami. A expressão mostrada em [6] foi obtida a partir de uma expressão obtida em [8] para a SEP do QAM retangular sob ruído AWGN. Um dos termos da expressão de [8] contém um produto de duas funções $\mathrm{Q}(x)$ e complica a avaliação da SEP sob a fdp do desvanecimento. A vantagem das expressões de Cho e Yoon é que a BEP sob ruído gaussiano é escrita como uma soma de funções $\operatorname{erfc}(x)$ e no presente trabalho essas funções são reescritas e expressas no intervalo de integração $[0, \pi / 2]$, que diminui consideravelmente o processo de avaliação numérica.

As aplicações dos esquemas de modulação QAM vão desde alguns modens com transmissão síncrona, que usam constelações 16-QAM, 64-QAM e 256-QAM, aos padrões de televisão digital DVB-T (Digital Video BroadcastingTerrestrial), ISDB-T (Integrated Services Digital Broadcasting Terrestrial) e ISDTV (Integrated Services Digital TV). No padrão ISDB-T os dados são codificados e arranjados em 
blocos de 188 bytes, chamados de MPEG2 TSP (Transport Stream Packet), são enviados para um segmentador e em seguida são arranjados em um, dois ou três blocos de codificação. Esses blocos são chamados de camadas e seus dados passam por moduladores e podem ser mapeados em símbolos das constelações 16-QAM ou 64-QAM.

No presente artigo, as expressões da BEP sob ruído AWGN obtidas em [5], $P(E \mid \gamma)$, são usadas para que se possa obter as novas expressões de BEP sob diferentes formas de desvanecimento por meio do cálculo da média

$$
P_{b}=\int_{0}^{\infty} P_{b}(E \mid \gamma) p_{\gamma}(\gamma ; \bar{\gamma}) d \gamma,
$$

em que $p_{\gamma}(\gamma ; \bar{\gamma})$ é a função densidade de probabilidade (fdp) univariada da relação sinal-ruído (SNR) instantânea e $\bar{\gamma}$ denota a SNR média. A avaliação dessa integral é feita, para os diferentes casos de desvanecimento considerados, expressando a função erro complementar $\operatorname{erfc}(x)$, dada por

$$
\operatorname{erfc}(x)=\frac{2}{\sqrt{\pi}} \int_{x}^{\infty} e^{-t^{2}} d t
$$

na forma

$\operatorname{erfc}(x)=2 \mathrm{Q}(\sqrt{2} x)=\frac{2}{\pi} \int_{0}^{\frac{\pi}{2}} \exp \left(-\frac{x^{2}}{\operatorname{sen}^{2} v}\right) d v, \quad x \geq 0$

e usando a função geratriz de momentos que aparece em todos os casos analisados. Em (3) a função $\mathrm{Q}(x)$ representa a probabilidade de que uma variável aleatória gaussiana de média nula e variância unitária tenha valor maior que $x$. Antes de prosseguir com a avaliação da integral dada em (1), a próxima seção apresenta o modelo do canal de transmissão e uma breve discussão sobre diferentes formas de desvanecimento que podem ser considerados.

\section{Modelo do CANal E Do Receptor}

No modelo de ambiente de propagação considerado, o sinal transmitido $s(t)$ é enviado por meio de $N$ subcanais com desvanecimento plano. Cada um desses subcanais forma um enlace estabelecido com uma das $N$ entradas de um receptor MRC, como mostrado na Fig. 1. Em cada um dos enlaces, o sinal transmitido sofre sucessivas variações de amplitude e fase, causadas pelos obstáculos que se encontram em seu percurso entre as antenas transmissora e receptora. Essas variações de amplitude e fase são comumente conhecidas como desvanecimento e são representadas, no $k$-ésimo enlace, por $\alpha_{k} e^{-j \theta_{k}}$.

Adicionalmente às variações de amplitude e fase, a propagação por múltiplos percursos provoca atrasos no sinal transmitido, os quais são denotados, para o $k$-ésimo enlace, por $\tau_{k}$. Devido à natureza da propagação em ambientes urbanos, é razoável supor que os parâmetros $\alpha_{k}, \theta_{k}$ e $\tau_{k}$ podem ser modelados por variáveis aleatórias mutuamente independentes [4].

Após sofrer o efeito do desvanecimeno no $k$-ésimo enlace, o sinal sofre, na entrada do receptor, o efeito do ruído aditivo, geralmente de natureza térmica. Esse ruído AWGN é modelado, na entrada de cada ramo do receptor, por uma variável aleatória

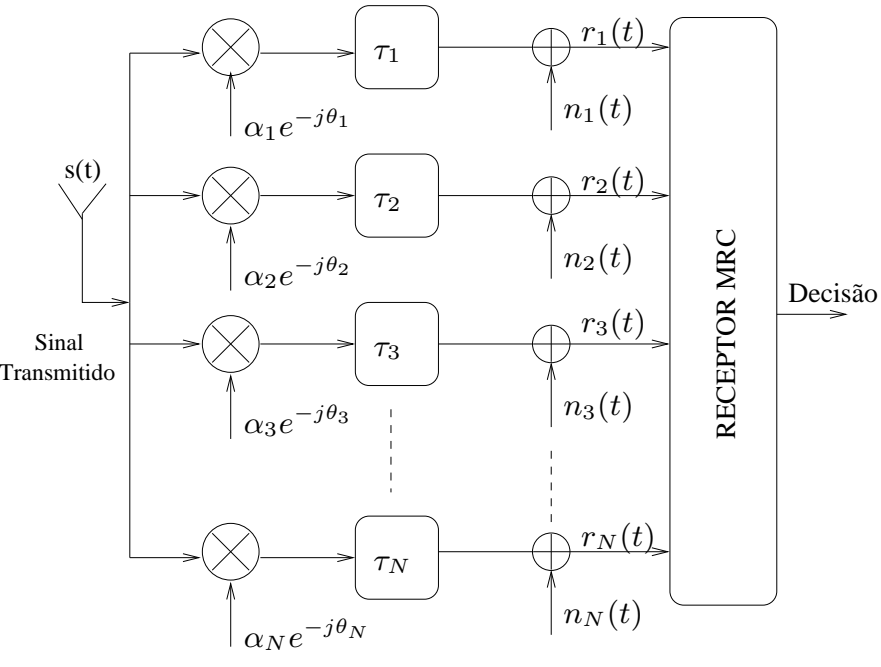

Fig. 1. Sistema de recepção coerente com diversidade.

gaussiana com densidade espectral de potência bilateral representada por $N_{0} / 2(\mathrm{~W} / \mathrm{Hz})$. Essas variáveis que modelam o ruído aditivo são consideradas mutuamente independentes nos $N$ ramos do receptor e independentes das variáveis aleatórias que modelam o efeito do desvanecimento.

O sinal transmitido, representado por $s(t)$, pode ser escrito como

$$
s(t)=\sum_{i=-\infty}^{\infty} S_{i} e^{-j \omega_{c} t} P_{T_{s}}\left(t-i T_{s}\right)
$$

em que $\omega_{c}$ é a freqüência da portadora, $P_{T_{s}}(\cdot)$ é um pulso retangular de duração $T_{s}$ e $S_{i}$ é o $i$-ésimo símbolo transmitido resultante do mapeamento de blocos de $K$ bits em uma das $M=2^{K}$ possíveis formas de onda a serem transmitidas. Admitindo que o receptor MRC tem perfeito conhecimento das atenuações e dos deslocamentos de fase no $k$-ésimo subcanal de transmissão, e que os símbolos são eqüiprováveis, podese escrever a razão sinal-ruído na saída do receptor MRC, $\gamma$, como

$$
\gamma=\frac{E_{s}}{N_{0}} \sum_{k=1}^{N} \alpha_{k}^{2}=\log _{2}(M) \frac{E_{b}}{N_{0}} \sum_{k=1}^{N} \alpha_{k}^{2}=\sum_{k=1}^{N} \gamma_{k},
$$

em que $\gamma_{k}=\log _{2}(M) \frac{E_{b}}{N_{0}} \alpha_{k}^{2}$ é a relação sinal-ruído por bit para cada ramo do receptor MRC.

A avaliação da integral dada em (1) pode ser realizada, a partir do resultado apresentado em (5), calculando a fdp da variável aleatória $\gamma$. Como a variável $\gamma$ é uma soma ponderada de $N$ variáveis independentes, $\alpha_{k}^{2}$, que dependem diretamente do tipo de desvanecimento presente no canal, é necessário antes calcular a fdp da $k$-ésima variável $\alpha_{k}^{2}$ para o tipo de desvanecimento analisado.

\section{CÁlculo da Probabilidade de Erro de Bit}

No caso de uma recepção multicanal, por meio de um receptor MRC com $N$ ramos, a fdp $p_{\gamma}(\gamma ; \bar{\gamma})$ em (1) passa a ser uma fdp multivariada representada por $p_{\gamma_{1}, \gamma_{2}, \cdots, \gamma_{N}}\left(\gamma_{1}, \gamma_{2}, \cdots, \gamma_{N}\right)$. Desse modo, a BEP mostrada 
em (1) passa a ser escrita como

$$
\begin{aligned}
P_{b} & =\int_{0}^{\infty} \int_{0}^{\infty} \cdots \int_{0}^{\infty} P_{b}(E \mid \gamma) \\
& \cdot p_{\gamma_{1}, \gamma_{2}, \cdots, \gamma_{N}}\left(\gamma_{1}, \gamma_{2}, \cdots, \gamma_{N}\right) d \gamma_{1} d \gamma_{2} \cdots d \gamma_{N} .
\end{aligned}
$$

Como as variáveis $\gamma_{k}$ são consideradas estatisticamente independentes, (6) pode ser simplificada para

$$
P_{b}=\prod_{k=1}^{N} \int_{0}^{\infty} P_{b}(E \mid \gamma) p_{\gamma_{k}}\left(\gamma_{k} ; \bar{\gamma}_{k}\right) d \gamma_{k}
$$

A avaliação da integral em (7) para os esquemas de modulação M-PAM, M-QAM e R-QAM segue a partir das expressões obtidas por Cho e Yoon para ruído AWGN.

\section{A. Probabilidade de Erro para a modulação M-PAM}

No caso do esquema M-PAM, a probabilidade de erro de bit $P(E \mid \gamma)$ sob ruído gaussiano aditivo foi obtida por [5] e é dada por

$$
P_{b}(E \mid \gamma)=\frac{1}{\log _{2}(M)} \sum_{j=1}^{\log _{2}(M)} P_{b}(j)
$$

em que a probabilidade de que o $j$-ésimo bit esteja em erro é dada por

$$
\begin{aligned}
P_{b}(j) & =\frac{1}{M} \sum_{i=0}^{\left(1-2^{-j}\right) M-1}\{w(i, j, M) \\
& \left.\cdot \operatorname{erfc}\left((2 i+1) \sqrt{\frac{3 \log _{2}(M) \gamma}{\left(M^{2}-1\right)}}\right)\right\}
\end{aligned}
$$

e os coeficientes $w(i, j, M)$ são dados por

$$
w(i, j, M)=(-1)^{\left\lfloor\frac{i 2^{j-1}}{M}\right\rfloor} \cdot\left(2^{j-1}-\left\lfloor\frac{i \cdot 2^{j-1}}{M}+\frac{1}{2}\right\rfloor\right) .
$$

Substituindo (8) e (9) em (7), tem-se, para um receptor com $N$ ramos e desvanecimento independente,

$$
\begin{aligned}
P_{b} & =\frac{1}{\log _{2}(M)} \int_{0}^{\infty} \int_{0}^{\infty} \cdots \int_{0}^{\infty} \sum_{j=1}^{\log _{2}(M)} P_{b}(j) \\
& \times \prod_{k=1}^{N} p_{\gamma_{k}}\left(\gamma_{k} ; \bar{\gamma}_{k}\right) d \gamma_{1} d \gamma_{2} \cdots d \gamma_{N} .
\end{aligned}
$$

Substituindo a expressão de $P_{b}(j),(9)$, em (11), tem-se

$$
\begin{aligned}
P_{b} & =\frac{1}{M \log _{2}(M)} \int_{0}^{\infty} \cdots \int_{0}^{\infty} \sum_{j=1}^{\log _{2}(M)} \sum_{i=0}^{\left(1-2^{-j}\right) M-1} w(i, j, M) \\
& \cdot \operatorname{erfc}\left((2 i+1) \sqrt{\frac{3 \log _{2}(M) \gamma_{k}}{\left(M^{2}-1\right)}}\right) \\
& \cdot \prod_{k=1}^{N} p_{\gamma_{k}}\left(\gamma_{k} ; \bar{\gamma}_{k}\right) d \gamma_{1} d \gamma_{2} \cdots d \gamma_{N} .
\end{aligned}
$$

Intercalando as integrais com os somatórios em (12), pode-se reescrever a expressão da probabilidade de erro na forma

$$
\begin{aligned}
P_{b} & =\frac{1}{M \log _{2}(M)} \sum_{j=1}^{\log _{2}(M)} \sum_{i=0}^{\left(1-2^{-j}\right) M-1} w(i, j, M) \\
& \cdot \int_{0}^{\infty} \int_{0}^{\infty} \cdots \int_{0}^{\infty} \operatorname{erfc}\left((2 i+1) \sqrt{\frac{3 \log _{2}(M) \gamma_{k}}{\left(M^{2}-1\right)}}\right) \\
& \cdot \prod_{k=1}^{N} p_{\gamma_{k}}\left(\gamma_{k} ; \bar{\gamma}_{k}\right) d \gamma_{1} d \gamma_{2} \cdots d \gamma_{N} .
\end{aligned}
$$

Como as variáveis $\gamma_{k}$ são independentes, a integral múltipla em (13) pode ser reescrita como um produto de $N$ integrais. Desse modo, $P_{b}$ pode ser reescrita como

$$
\begin{aligned}
P_{b} & =\frac{1}{M \log _{2}(M)} \sum_{j=1}^{\log _{2}(M)} \sum_{i=0}^{\left(1-2^{-j}\right) M-1} w(i, j, M) \\
& \cdots \prod_{k=1}^{N}\left[\int_{0}^{\infty} \operatorname{erfc}\left((2 i+1) \sqrt{\frac{3 \log _{2}(M) \gamma_{k}}{\left(M^{2}-1\right)}}\right)\right. \\
& \left.\cdot p_{\gamma_{k}}\left(\gamma_{k} ; \bar{\gamma}_{k}\right) d \gamma_{k}\right] .
\end{aligned}
$$

Fazendo $a_{i}(M)=\frac{3(2 i+1)^{2} \log _{2}(M)}{\left(M^{2}-1\right)}$ e usando a representação de $\operatorname{erfc}(x)$ dada em (3), pode-se reescrever $P_{b}$ na forma

$$
\begin{aligned}
P_{b} & =\frac{1}{M \log _{2}(M)} \sum_{j=1}^{\log _{2}(M)} \sum_{i=0}^{\left(1-2^{-j}\right) M-1} w(i, j, M) \\
& \times \prod_{k=1}^{N}\left[\frac{2}{\pi} \int_{0}^{\frac{\pi}{2}} \int_{0}^{\infty} \exp \left(-\frac{a_{i}(M) \gamma_{k}}{\operatorname{sen}^{2}(v)}\right) p_{\gamma_{k}}\left(\gamma_{k} ; \bar{\gamma}_{k}\right) d \gamma_{k} d v\right] .
\end{aligned}
$$

Comparando a integral imprópria interna em (15) com a função geratriz de momentos (MGF - Moment Generating Function) de uma variável aleatória $X$ positiva, dada por

$$
M_{X}(s)=\int_{0}^{\infty} e^{s x} p_{X}(x) d x
$$

percebe-se que essa integral é a MGF da variável $\gamma_{k}$ avaliada em $s=-\frac{a_{i}(M)}{\operatorname{sen}^{2}(v)}$. Como a fdp de $\gamma_{k}$ também está escrita em função da SNR média por bit, a MGF de $\gamma_{k}$ é denotada por $M_{\gamma_{k}}\left(-\frac{a_{i}(M)}{\operatorname{sen}^{2}(v)} ; \bar{\gamma}_{k}\right)$. Desse modo, a BEP do esquema M-PAM pode ser escrita em termos da MGF como

$$
\begin{aligned}
P_{b} & =\frac{1}{M \log _{2}(M)} \sum_{j=1}^{\log _{2}(M)} \sum_{i=0}^{\left(1-2^{-j}\right) M-1} w(i, j, M) \\
& \times \prod_{k=1}^{N}\left[\frac{2}{\pi} \int_{0}^{\frac{\pi}{2}} M_{\gamma_{k}}\left(-\frac{a_{i}(M)}{\operatorname{sen}^{2}(v)} ; \bar{\gamma}_{k}\right) d v\right]
\end{aligned}
$$

Assumindo que o ruído é identicamente distribuído nos $N$ subcanais e que a SNR média por bit na entrada dos $k$ ramos do receptor MRC, $\bar{\gamma}_{k}$, é a mesma em todos os ramos e vale $\bar{\gamma}$, a probabilidade de erro de bit dada em (17) pode ser reescrita 
como

$$
\begin{aligned}
P_{b} & =\frac{1}{M \log _{2}(M)} \sum_{j=1}^{\log _{2}(M)} \sum_{i=0}^{\left(1-2^{-j}\right) M-1} w(i, j, M) \times \\
& {\left[\frac{2}{\pi} \int_{0}^{\frac{\pi}{2}} M_{\gamma}\left(-\frac{a_{i}(M)}{\operatorname{sen}^{2}(v)} ; \bar{\gamma}\right) d v\right]^{N} . }
\end{aligned}
$$

\section{B. Probabilidade de Erro para a Modulação $M-Q A M$}

Em relação à modulação M-QAM, a expressão obtida em [5] para o cálculo da probabilidade de erro de bit sob ruído AWGN, condicionada à SNR, $P(E \mid \gamma)$, é dada por

$$
P_{b}(E \mid \gamma)=\frac{1}{\log _{2} \sqrt{M}} \sum_{j=1}^{\log _{2} \sqrt{M}} P_{b}(j)
$$

em que a probabilidade de erro do $j$-ésimo bit, $P_{b}(j)$, é dada por

$$
\begin{aligned}
P_{b}(j) & =\frac{1}{\sqrt{M}} \sum_{i=0}^{\left(1-2^{-j}\right) \sqrt{M}-1}\{w(i, j, M) \\
& \left.\times \operatorname{erfc}\left((2 i+1) \sqrt{\frac{3 \log _{2} M \gamma}{2(M-1)}}\right)\right\}
\end{aligned}
$$

e os pesos $w(i, j, M)$ são dados por

$$
w(i, j, M)=(-1)^{\left\lfloor\frac{i 2^{j-1}}{\sqrt{M}}\right\rfloor} \cdot\left(2^{j-1}-\left\lfloor\frac{i \cdot 2^{j-1}}{\sqrt{M}}+\frac{1}{2}\right\rfloor\right) .
$$

A partir desses resultados, a BEP para a modulação M-QAM sob o efeito do desvanecimento, para o $k$-ésimo ramo do receptor é obtida por meio da integral

$$
\begin{aligned}
P_{b} & =\frac{1}{\sqrt{M} \log _{2}(\sqrt{M})} \sum_{j=1}^{\log _{2}(\sqrt{M})} \sum_{i=0}^{\left(1-2^{-j}\right) \sqrt{M}-1} w(i, j, M) \\
& \cdot \int_{0}^{\infty} \int_{0}^{\infty} \cdots \int_{0}^{\infty} \operatorname{erfc}\left((2 i+1) \sqrt{\frac{3 \log _{2}(M) \gamma_{k}}{2(M-1)}}\right) \\
& \cdot \prod_{k=1}^{N} p_{\gamma_{k}}\left(\gamma_{k} ; \bar{\gamma}_{k}\right) d \gamma_{1} d \gamma_{2} \cdots d \gamma_{k} .
\end{aligned}
$$

Fazendo $a_{i}(M)=\frac{3(2 i+1)^{2} \log _{2}(M)}{2(M-1)}$ e usando a expressão de $\operatorname{erfc}(x)$ apresentada em (3), tem-se

$$
\begin{aligned}
P_{b} & =\frac{1}{\sqrt{M} \log _{2}(\sqrt{M})} \sum_{j=1}^{\log _{2}(\sqrt{M})} \sum_{i=0}^{\left(1-2^{-j}\right) \sqrt{M}-1} w(i, j, M) \\
& \cdot \prod_{k=1}^{N}\left[\frac{2}{\pi} \int_{0}^{\frac{\pi}{2}} \int_{0}^{\infty} \exp \left(-\frac{a_{i}(M) \gamma_{k}}{\operatorname{sen}^{2} v}\right) p_{\gamma_{k}}\left(\gamma_{k} ; \bar{\gamma}_{k}\right) d \gamma_{k} d v\right] .
\end{aligned}
$$

Em termos da função geratriz de momentos, $P_{b}$ pode ser reescrita como

$$
\begin{aligned}
P_{b} & =\frac{1}{\sqrt{M} \log _{2}(\sqrt{M})} \sum_{j=1}^{\log _{2}(\sqrt{M})} \sum_{i=0}^{\left(1-2^{-j}\right) \sqrt{M}-1} w(i, j, M) \\
& \cdot \prod_{k=1}^{N}\left[\frac{2}{\pi} \int_{0}^{\frac{\pi}{2}} M_{\gamma_{k}}\left(-\frac{a_{i}(M)}{\operatorname{sen}^{2} v} ; \bar{\gamma}_{k}\right) d v\right] .
\end{aligned}
$$

Se for assumido que o desvanecimento é identicamente distribuído e que a SNR média por bit é a mesma em todos os ramos do receptor MRC, a expressão de $P_{b}$ passa a ser escrita como

$$
\begin{aligned}
P_{b} & =\frac{1}{\sqrt{M} \log _{2}(\sqrt{M})} \sum_{j=1}^{\log _{2}(\sqrt{M})} \sum_{i=0}^{\left(1-2^{-j}\right) \sqrt{M}-1} w(i, j, M) \\
& \times\left[\frac{2}{\pi} \int_{0}^{\frac{\pi}{2}} M_{\gamma}\left(-\frac{a_{i}(M)}{\operatorname{sen}^{2} v} ; \bar{\gamma}\right) d v\right]^{N} .
\end{aligned}
$$

\section{PRobabilidade DE ERro PARA o ESQUEMA R-QAM}

Em relação ao esquema R-QAM, também denotado $I \times J$ QAM, a expressão para a probabilidade de erro de bit sob ruído gaussiano branco e mapeamento Gray nos bits da constelação com $I \times J$ símbolos, dada a $\operatorname{SNR} \gamma, P(E \mid \gamma)$, é dada por

$$
P_{b}(E \mid \gamma)=\frac{1}{\log _{2}(I \cdot J)}\left(\sum_{l=1}^{\log _{2} I} P_{I}(l)+\sum_{n=1}^{\log _{2} J} P_{J}(n)\right),
$$

em que as probabilidades $P_{I}(l)$ e $P_{J}(j)$ são dadas respectivamente por

$$
\begin{gathered}
P_{I}(l)=\frac{1}{I} \sum_{i=0}^{\left(1-2^{-l}\right) I-1}\left\{w(i, l, I) \operatorname{erfc}\left(\sqrt{a_{i}(I, J) \gamma}\right)\right\} \\
P_{J}(n)=\frac{1}{J} \sum_{j=0}^{\left(1-2^{-n}\right) J-1}\left\{w(j, n, J) \operatorname{erfc}\left(\sqrt{a_{j}(I, J) \gamma}\right)\right\}
\end{gathered}
$$

e os pesos $w(i, l, I)$ e $w(j, n, J)$ podem ser obtidos, a partir de (10), substituindo os índices $i, l, I$ e $j, n, J$, nesta mesma ordem, nos índices $i, j, M$. Os termos $a_{i}(I, J)$ e $a_{j}(I, J)$ são obtidos a partir da equação

$$
a_{i}(I, J)=\frac{3(2 i+1)^{2} \log _{2}(I \cdot J)}{I^{2}+J^{2}-2} .
$$

A avaliação da probabilidade de erro de bit do esquema R-QAM pode ser realizada de modo similar ao utilizado para os esquemas de modulação M-PAM e M-QAM. Para facilitar o desenvolvimento, pode-se separar (26) em duas equações, denotadas por $P_{b_{I}}(E \mid \gamma)$ e $P_{b_{J}}(E / \gamma)$. Substituindo 
as expressões $P_{I}(l)$ e $P_{J}(n)$ nessas expressões, obtém-se

$$
\begin{aligned}
P_{b_{I}}(E \mid \gamma) & =\frac{1}{I \log _{2}(I \cdot J)} \sum_{l=1}^{\log _{2} I} \sum_{i=0}^{\left(1-2^{-l}\right) I-1} w(i, l, I) \\
\cdot & \operatorname{erfc}\left(\sqrt{a_{i}(I, J) \gamma}\right), \\
P_{b_{J}}(E \mid \gamma) & =\frac{1}{J \log _{2}(I \cdot J)} \sum_{n=1}^{\log _{2} J} \sum_{j=0}^{J\left(1-2^{-n}\right) J-1} w(j, n, J) \\
& \cdot \operatorname{erfc}\left(\sqrt{a_{j}(I, J) \gamma}\right) .
\end{aligned}
$$

A média das expressões $P_{b_{I}}\left(E \mid \gamma_{k}\right)$ e $P_{b_{J}}\left(E \mid \gamma_{k}\right)$, a partir da integral dada em (6), sob consideração de desvanecimento independente nos $N$ ramos do receptor MRC, considerando a forma alternativa da função $\operatorname{erfc}(x)$ dada em (3), leva às expressões

$$
\begin{aligned}
P_{b_{I}}= & \frac{1}{I \log _{2}(I \cdot J)} \sum_{l=1}^{\log _{2} I} \sum_{i=0}^{\left(1-2^{-l}\right) I-1} w(i, l, I) \\
& \cdot \prod_{k=1}^{N}\left[\frac{2}{\pi} \int_{0}^{\frac{\pi}{2}} M_{\gamma_{k}}\left(-\frac{a_{i}(I, J)}{\operatorname{sen}^{2} v} ; \bar{\gamma}_{k}\right) d v\right] \\
P_{b_{J}}= & \frac{1}{J \log _{2}(I \cdot J)} \sum_{n=1}^{\log _{2} J} \sum_{j=0}^{\left(1-2^{-n}\right) J-1} w(j, n, J) \\
& \cdot \prod_{k=1}^{N}\left[\frac{2}{\pi} \int_{0}^{\frac{\pi}{2}} M_{\gamma_{k}}\left(-\frac{a_{j}(I, J)}{\operatorname{sen}^{2} v} ; \bar{\gamma}_{k}\right) d v\right] .
\end{aligned}
$$

Considerando que o desvanecimento seja identicamente distribuído nos $N$ subcanais do modelo de sistema de comunicação considerado, as novas expressões de $P_{b_{I}}$ e $P_{b_{j}}$ passam a ser escritas como

$$
\begin{aligned}
P_{b_{I}}= & \frac{1}{I \log _{2}(I \cdot J)} \sum_{l=1}^{\log _{2} I\left(1-2^{-l}\right) I-1} \sum_{i=0}^{\frac{\pi}{2}} w(i, l, I) \\
\cdot & {\left[\frac{2}{\pi} \int_{0}^{\frac{a_{i}}{2}} M_{\gamma}\left(-\frac{\left.a_{i} J\right)}{\operatorname{sen}^{2} v} ; \bar{\gamma}\right) d v\right]^{N} } \\
P_{b_{J}}= & \frac{1}{J \log _{2}(I \cdot J)} \sum_{n=1}^{\log _{2} J\left(1-2^{-n}\right) J-1} \sum_{j=0} w(j, n, J) \\
\cdot & {\left[\frac{2}{\pi} \int_{0}^{\frac{\pi}{2}} M_{\gamma}\left(-\frac{a_{j}(I, J)}{\operatorname{sen}^{2} v} ; \bar{\gamma}\right) d v\right]^{N} . }
\end{aligned}
$$

A probabilidade de erro resultante $P_{b}$ é então obtida pela soma das probabilidades $P_{b_{I}}$ e $P_{b_{J}}$. Como se pode perceber pelas equações obtidas para os três esquemas de modulação, as expressões de BEP são dadas todas em termos da função geratriz de momentos da SNR instantânea $\gamma$. Essa função varia, de acordo com a fdp de $\gamma$, para cada distribuição de desvanecimento considerada. Algumas distribuições comumente usadas em ambientes móveis e que podem ser empregadas no estudo feito nesse artigo são as distribuições Rayleigh, Hoyt, Rice, Nakagami e sombreamento Log-Normal.

\section{CONCLUSÃO}

Neste trabalho foram apresentadas expressões exatas para o cálculo da probabilidade de erro de bits de um modelo de sistema de comunicação multicanal, usando os esquemas de modulação M-PAM, M-QAM e R-QAM sob desvanecimento generalizado. $\mathrm{Na}$ abordagem utilizada, a expressão da função erro complementar foi reescrita em termos de uma integral definida no intervalo $[0, \pi / 2]$. As expressões obtidas para a BEP, por ramo do receptor, em função da SNR média por ramo, $\bar{\gamma}_{k}$, foram expressas em termos da integral da MGF da SNR instantânea, $M_{\gamma}(t ; \bar{\gamma})$, no intervalo $[0, \pi / 2]$. A vantagem dessa abordagem é que ela pode ser aplicada a qualquer tipo de desvanecimento, e as integrais, mesmo que não forneçam expressões exatas, podem ser avaliadas numericamente.

\section{REFERÊNCIAS}

[1] C. Kim, Y. Kim, G. Jeong, and H. Lee. BER analysis of QAM with MRC space diversity in Rayleigh fading channels. In in Proc. IEEE Int. Symp. Personal, Indoor and Mobile Communications (PIMRC'95), pages 482-485, Toronto, ON, Canada, September 1995.

[2] J. Lu, T. T. Tjhung, and C. C. Chai. Error probability performance of L-branch diversity reception of MQAM in Rayleigh fading. IEEE Transactions on Communications, 46:179-181, February 1998.

[3] G. Femenias and I. Furió. Dual MRC diversity reception of TCM-MPSK signals over Nakagami fading channels. Electronics Letters, 32:17521754, September 1996.

[4] M. S. Alouini and A. J. Goldsmith. A unified approach for calculating error rates of linearly modulated signals over generalized fading channels. IEEE Transactions on Communications, 47(9):1324-11334, September 1999.

[5] K. Cho and D. Yoon. On the general BER expression of one and two dimensional amplitude modulations. IEEE Transactions on Communications, 50(7):1074-1080, 2002.

[6] G. K. Karagiannidis. On the symbol error probability of general order rectangular QAM in Nakagami-m fading. IEEE Communications Letters, 10(11):745-747, November 2006.

[7] N. C. Beaulieu and Y. Chen. Closed-form expressions for the exact symbol error probability of 32-Cross-QAM in AWGN and in slow Nakagami fading. IEEE Communications Letters, 11(4):310-312, April 2007.

[8] N. C. Beaulieu. A useful integral for wireless communication theory and its applications to rectangular signaling constellation error rates. IEEE Transactions Communications, 54(5):802-805, May 2006.

[9] J. G. Proakis. Digital Communications. McGraw-Hill, New York, 3 edition, 1995.

[10] M. Abramowitz and I. A. Stegun. Handbook of Mathematical Functions. Dover publications, Inc., New York, 1970.

[11] I. S. Gradshteyn and I. M. Ryzhik. Table of Integrals, Series and Products. CA Academic, 5ht ed., San Diego, 1994.

[12] J. Lu, K. B. Lefaief, J. C. Chuang, and M. L. Liou. M-PSK and M-QAM BER computation using signal-space concepts. IEEE Transactions on Communications, 47(2):181-184, 1999.

[13] L. L. Yang and L. Hanzo. A recursive algorithm for error probability evaluation of M-QAM. IEEE Communications Letters, 4(10):304-306, 2000.

[14] P. M. Fortune, L. Hanzo, and R. Steele. On the computation of 16QAM and 64-QAM performance in Rayleigh-fading channels. IEICE Transactions on Communications, E75B(6):466-475, 1992.

[15] M. G. Shayesteh and A. Aghamohammadi. On the error probability of linearity of modulated signals on frequency-flat Ricean, Rayleigh and AWGN channels. IEEE Transactions on Communications, 43(2):14541466, 1995.

[16] K. Pomorski. Gauss-Hermite approximation formula. Computer Physics Communications, 174(3):181-186, February 2006.

[17] A. Conti, M. Z. Win, and M. Chiani. Slow adaptive M-QAM with diversity in fast fading and shadowing. IEEE Transactions Communications, 55(5):895-905, May 2007. 